\title{
Fuel Economy of a Current Hybrid London Bus and Fuel Cell Bus Application Evaluation
}

\author{
Cedrick Lin \\ University College London, UK \\ cheng-che.lin.11@ucl.ac.uk
}

\author{
Dr. Julius Partridge \\ University College London, UK \\ julius.partridge.09@ucl.ac.uk
}

\author{
Prof. Richard Bucknall \\ University College London, UK \\ r.bucknall@ucl.ac.uk
}

\begin{abstract}
London has over 8,500 buses in operation, carrying six million passengers on 700 routes each day. In central London the majority of the bus fleet has been replaced by diesel-electric hybrid buses. In this study, we will investigate the degree of energy efficiency via practical on-road bus performance recordings, forming a foundation for future improvements to diesel and fuel cell hybrid bus design. Research at UCL has investigated the design and performance of the ENVIRO $400 \mathrm{H}$ model bus on various different routes in London, obtaining a wide range of data for real world performance. This data includes information on routes, usage, energy consumption and passenger count profiling. Analysis has been conducted on the efficiency of the propulsion system over all the data sets. This knowledge can be used as the basis for developing computer modelling capabilities to in the future to optimize the system performance. The key components in the propulsion system are the diesel engine, generator, converter, battery bank, and traction motor. The energy management strategy has been analysed for different operating conditions and will be discussed in this paper. It was concluded that the system performance varied, with a number of patterns emerging with regards to the engine load and battery State of Charge for providing the propulsion power requirements. The operation strategies employed have been analysed to give a detailed understanding of the operation of the diesel-electric hybrid propulsion system under real-world operation.
\end{abstract}

Index Terms-- Electric vehicles, Energy management, Fuel cells, Fuel economy, Propulsion.

\section{INTRODUCTION}

Reducing greenhouse gas emissions, especially $\mathrm{CO} 2$, has been an issue for many countries. The proportion of emissions is divided by sectors and, according to the IEA annual report (2010), transportation makes up $22 \%$ of total $\mathrm{CO} 2$ emission globally [1]. Of all means of transportation, buses are of great interest in this study owing to their operation and usage. The requirements demanded by Transport for London (TfL) dictate that a bus has to be in operation for over 18 hours a day without need for refuelling or maintenance. In addition, unlike trains, buses are flexible to their routes and are therefore capable of running off the expected route. The mechanical behaviour of a bus is also subject to highly variable task demand in aspects such as frequent stop-and-start, passenger weight change, etc. The National Travel Survey 2014 depicts that the mileage covered by buses in London has increased in the last twenty years by $58 \%$ [2]. Both of these characteristics provide opportunities for buses to be used as a research subject. In other words, the analysis can be applied to similar modes of transportation such as automobiles. In 2006 the first hybrid bus was put into operation in London on route 360 and soon after more hybrid electric buses were put into operation in London. In 2015 there were 1,500 hybrid buses, 22 electric buses, and 8 hydrogen buses [3]. They successfully achieved a 30\% reduction in emissions through the hybrid bus scheme with continued improvements being made. The technologies in use here are threefold: hybrid electric technology, pure electrical energy storage system (ESS) technology, and fuel cell hybrid system technology. To be more precise, these are a hybrid electric vehicle (HEV) with internal combustion engine (ICE), an electric vehicle (EV) running purely with energy storage, and a fuel cell vehicle (FCV) [4]. The major components include an ICE, FC, battery, and supercapacitor. Theoretically speaking infinite energy can be supplied by an ICE or FC, provided there is an infinite fuel supply; however, this is limited by the amount of fuel that can be stored on board. On the other hand, the battery and supercapacitor can only carry energy up to their design limit. As for the performance characteristics of each of the technologies, the Ragone diagram in Figure 1 details the energy density with respect to power density [5]. It demonstrates the energy (Wh) stored per unit weight $(\mathrm{kg})$, for each technology. ICEs have good energy and power performance, making them difficult to replace as they are well suited to the demands of bus operation. Fuel cells are suited to producing stable electrical power output but perform poorly when handling peak transient power requirements. On the other hand batteries can provide the required transient response in seconds and supercapacitors in microseconds. The

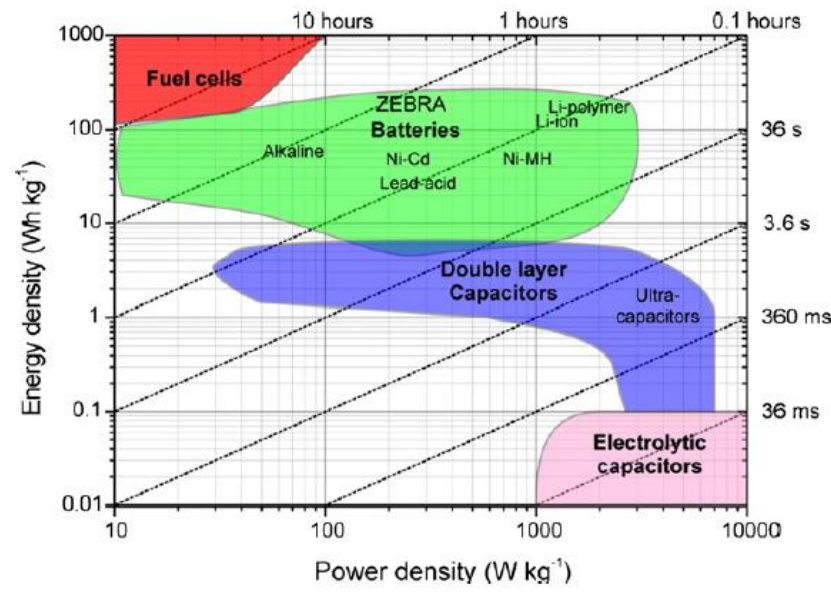

Figure 1 Ragone plot describing energy storage technologies in terms of energy density and power density. Diagonal perforated lines represent different characteristic times. [5] 
drawbacks of batteries are their short cycle life and long charging time. Conversely supercapacitors have longer cycle life of over 500,000 times [6] with very short charge/discharge times. However, they are not well suited to storing large amounts of energy. To summarise, a fuel cell with adequate ESS could be a better solution to manage both constant and peak transient power demands.

With these technologies, integrated systems can be compared through appropriate simulations. In order to provide fair comparison of the economics and performance of these technologies a reference baseline needs to be defined University College London and University of Sheffield have a joint project, HyFCap, which includes academic groups of chemical, civil, and mechanical engineering. The aim of the project is to investigate the possibility of reducing the cost of a fuel cell hybrid bus through downsizing the size of the fuel cell and improved supercapacitors. UCL has purchased one of the ENVIRO $400 \mathrm{H}$ diesel electric hybrid buses manufactured by Alexander Dennis Ltd in 2013. This is a double decker bus and operates daily on route 388 . The propulsion system is a series hybrid composed of an ICE, generator, lithium-ion battery, traction motor, and the required power electronics [7]. We will use this as a baseline for comparing the different technologies in this study. Data mining of the performance of the bus has been carried out, with many iterations of road running data collection conducted. Meanwhile, hydrogen technology on buses is also being analysed with some examples already in operation. The London fuel cell bus is a series hybrid bus, comprised of a hydrogen fuel cell, supercapacitor, and power electronics, operating on the route RV1. However, information at this time is limited since direct data acquisition has not been carried out. In this study, several hybrid fuel cell systems will be modelled using MATLAB/Simulink with the aims of comparing and analysing both performance and economics.

This paper will first present the methodology of data collection, experimental results, result processing, validation, analysis, results and discussion, computer modelling, hydrogen buses, future work and conclusions.

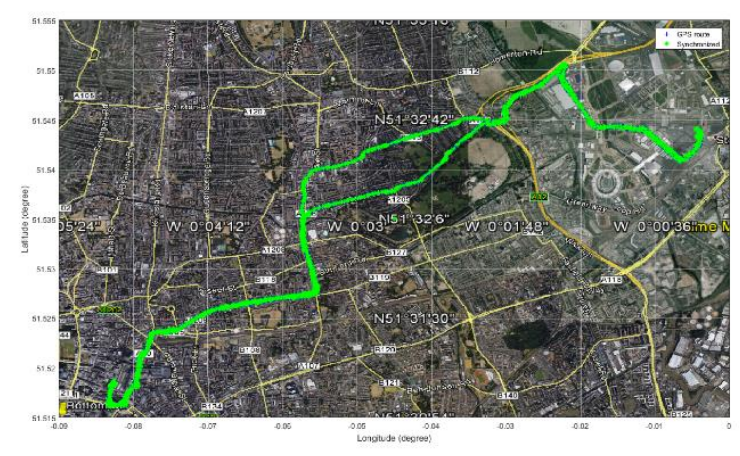

Figure 2 Second iteration. Route is between Liverpool Street and Stratford on 2nd March 2015 [8].

\section{METHODOLOGY}

\section{A. DATA COLLECTION}

In order to understand the real power requirements of buses operating in London, field research of various scenarios has been conducted with data collected from three sources.

The first step was to identify the information needed to assess the performance of the bus. The performance of the power system is the foundation of this research. To assess this the internal behaviour of the system components needs to be considered, such as the driver's command, engine speed, engine torque, generator power, battery current, battery voltage, bus voltage, motor power, auxiliary power, and fuel rate. In addition, an independent GPS recorder provided realtime location data to record the bus route and determine the elevation change over the bus route. Finally, a video camera positioned on the front panel of the bus, recorded traffic conditions, providing a picture of the conditions under which the bus was operating.

The second step was to consider the operating conditions that were to be investigated, for example: changes in speed, road gradient, changes in overall mass and corresponding traffic conditions.

Finally three iterations of data were collected. These were conducted in the year of 2014 and 2015 on different routes. The detail of these tests are shown in Table 1 and discussed in the next section. The first iteration was the longest one and the only one collected by our own external data logger via protocol J1939 which is often used on heavy duty vehicles [9]. However, due to the lack of precision of some data sets, especially State-of-Charge of the battery, the whole data set was deemed to lack the necessary detail. The remaining two tests were successful and were supplemented with GPS/Video and weight counting. Although the test on route 388 contained the road conditions required for analysis a specifically designed route was utilised to obtain data for specific operating conditions. It focused on uphill and downhill operation to investigate high power demand and regenerative braking behaviour.

Unfortunately there was a serious error with the data collected on this iteration.

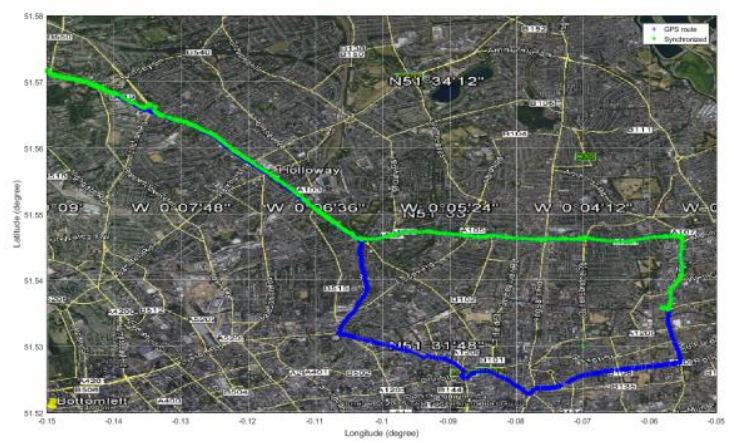

Figure 3 Third iteration. Route is designed to encounter loads of uphill/downhill. The blue line shows the entire journey and the green line shows the locations where have performance recording [8]. 
Table 1

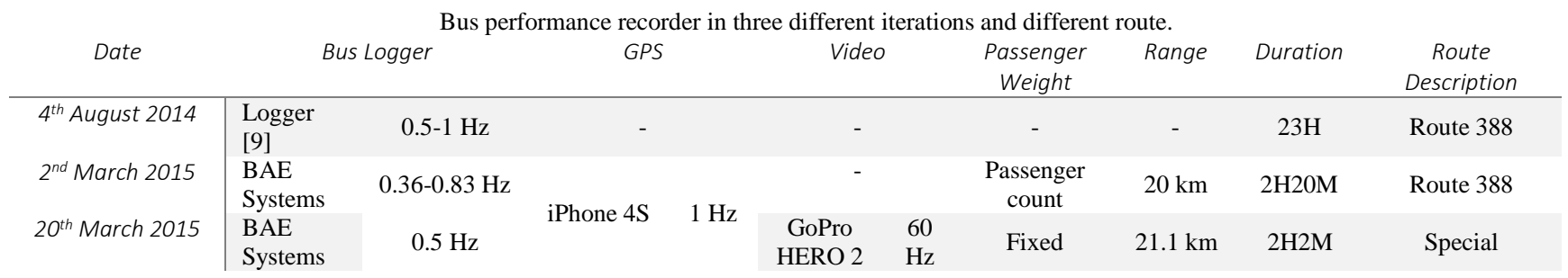

The bus performance data did not cover the whole period of the test and it resulted in a large portion of data being unavailable. As a whole, there was a problem with synchronising the timing of the data sets. Hence, a process was required in order to resolve the problem.

\section{B. DATA PROCESSING}

The original data was not consistent with SI units, therefore they were imported into MATLAB and then converted to equivalent sets of data for all iterations. When integrating the GPS data into the data sets there was a major problem in that the recording frequencies were not consistent between the different datasets. In other words, the time of the GPS data did not align to the time of the bus performance data. Consequently, a program that resolved this problem was applied. The method involved first finding the starting time point of the bus performance and GPS data and then interpolating the GPS time data with the time from the bus performance data. While doing this a search for large time gaps within each of the data sets was carried out and any significant time gaps were skipped. It is worth noting that the frequency of data recording for the data logger was not constant. For the second iteration the time between data points followed a cycle of $1.8 \mathrm{~s}, 1.8 \mathrm{~s}$, and $2.4 \mathrm{~s}$ between data points; the third iteration however, maintained a time between data points of $2 \mathrm{~s}$. In addition, the GPS entry had a constant frequency of $1 \mathrm{~Hz}$. As for video, the purpose was to use it as a reference when certain periods of performance were being examined. The corresponding footage can offer specific observations on the behaviour of the bus and allowed interpretation of the performance. The mass of the bus was calculated using the formula below:

$$
\begin{array}{r}
\text { Bus Weight }=12,000 \mathrm{~kg}(\text { unladen })+ \\
\text { Passenger number } \times 63 \mathrm{~kg}
\end{array}
$$

Elevation data was obtained through Google Maps API [8]. By sending a position in the form of longitude and latitude, the corresponding elevation data, under protocol 'json', is returned. The only restriction was that the web service has a daily entry limit of 250 sets of data and 1500 entries for each set. In this study, energy changes of the bus were a major concern that requires both the kinetic energy and potential energy of the vehicle. Kinetic and potential energy were calculated by equations (2) and (3), respectively.

Kinetic Energy $=\frac{1}{2} m v^{2}$
Potential Energy $=m g h$

where $\mathrm{m}$ is the bus weight, $\mathrm{v}$ is the bus velocity, $\mathrm{g}$ is the gravitational constant, and $\mathrm{h}$ is the elevation above sea level.

Finally, all the data sets were synchronized into one stream in MATLAB to complete further processing and generate results for analysis. Figure 4 demonstrates some of the available data streams and shows a 300 s period from which results and analysis can be drawn.
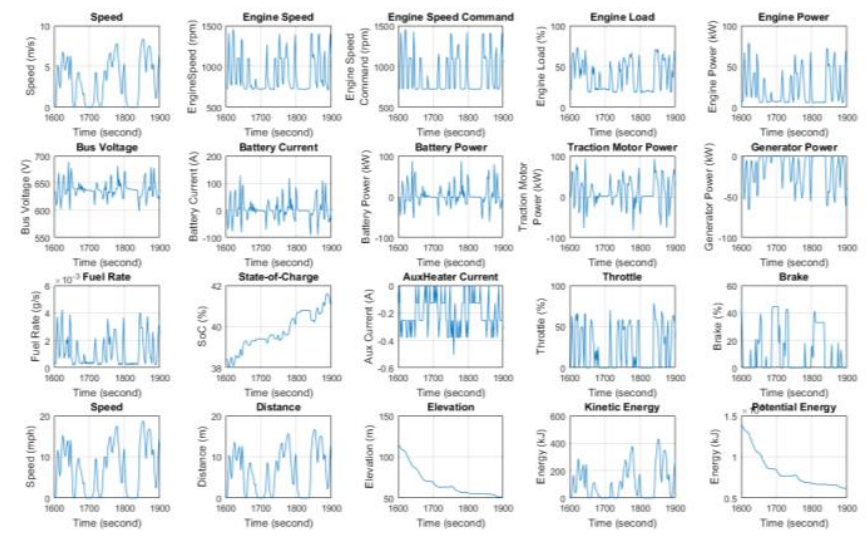

Figure 4 Bus performance shown in figure during 1600-1900 seconds of the special route. The bottom rows are information calculated not obtained from the bus directly.

\section{RESULTS AND DISCUSSION}

The validation of the data was completed by choosing short periods of time (10-20 seconds) and then observing the correlation between the data sets. In the case of the engine, engine power must follow fuel flow. In the case of the battery, battery power equals bus voltage multiplied by battery current. In addition, the power flow recorded for each component was compared to the component power rating to confirm that they operated within the specified limits. Then, with regards to the real traffic conditions shown in the video, the data was determined to be useful after all the relationships were checked.

In order to observe the behaviour of the bus, two approaches were considered. The first is a dynamic view, providing a direct image of the system response and power flow. The second is overall energy efficiency during different mode of operation and gives a more holistic view of the system performance. Figure 5, Figure 6, and Figure 7 show results for the same time period as Figure 4. Figure 5 shows vehicle speed, fuel rate, distance travelled, kinetic energy and potential energy to satisfy the energy demand required to propel the bus. The 
potential energy did not change significantly due to the small elevation change. Kinetic energy on the other hand accounted for a substantial amount of energy with a peak KE of the bus of $430 \mathrm{~kJ}$. This varied constantly and significantly which reflects the dynamic system behaviour. The behaviour of the internal components to meet the requirements is shown in Figure 6 and Figure 7. It can be observed that electricity is not delivered by the generator while the engine is idling, such as at 1700s, which led to significant loss of energy. In Figure 7, when the motor load is large, energy is delivered by both the generator and battery (1750s). Alternatively, as the vehicle decelerates, motor regenerative energy is delivered to the battery ESS. Further results showing the energy flow under different scenarios such as flat surface acceleration and downhill deceleration are presented. Within the selected period, integrating the power with respect to time derives the energy consumed/generated. By doing this the energy flow for different operations can be expressed in energy flow diagrams. These describe the direction and the magnitude of the energy flow in terms of the breadth of the arrow. Figure 8 and Figure 9 demonstrate the energy flow during two modes of operation. The percentages in red denote the proportion of energy compared to the total energy input into the system for both energy sources and sinks. The percentages in blue denote the conversion efficiency of each component and the percentages in black denote the energy loss in that component. Surprisingly large losses at the engine-generator set were discovered. It was found that the efficiency was significantly higher for acceleration than deceleration due to the increased load. The motor and mechanical efficiencies are yet to be determined.

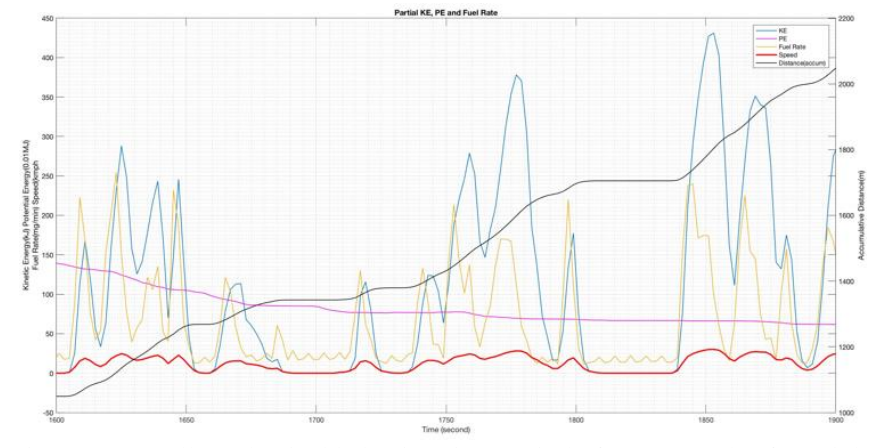

Figure 5 Overall mechanical behaviour in a period of 300 seconds of special route. Kinetic energy, potential energy, speed, distance travelled, and fuel rate are shown.

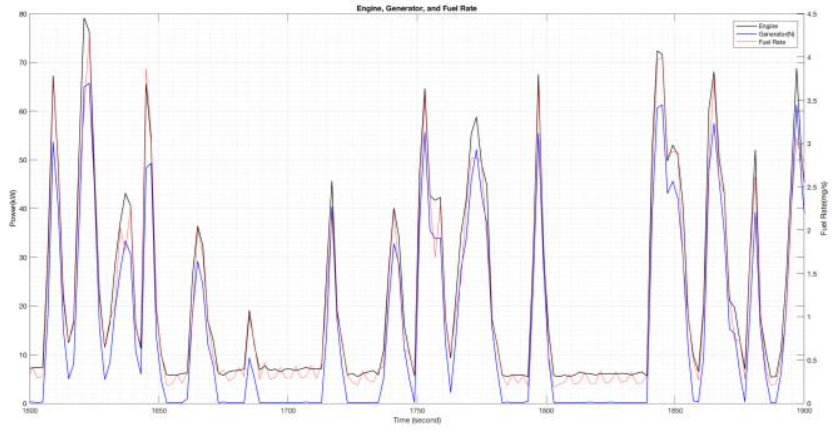

Figure 6 Engine, generator, and fuel rate in a period of 300 seconds.

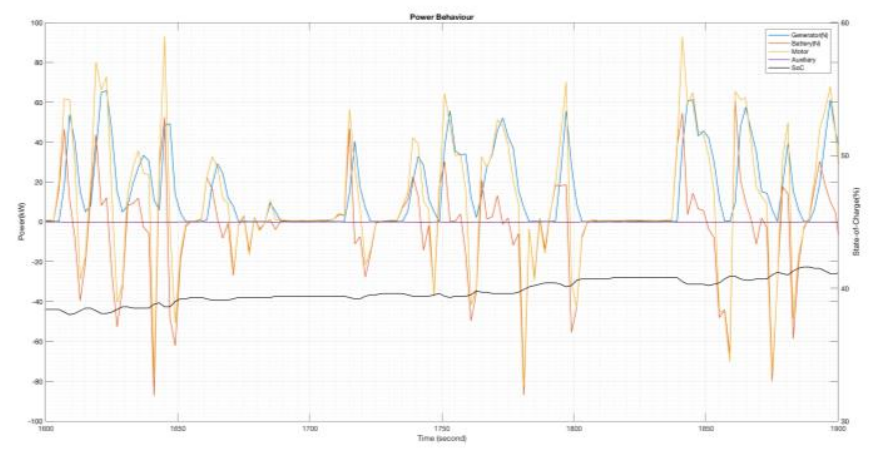

Figure 7 Power behaviour as in generator, battery, motor, auxiliary and SoC of battery in a period of 300 seconds.

In summary, the results have been presented to show both the instantaneous behaviour and energy flow for different modes of operation. The correlation between the behaviour of different components under various operating conditions has been investigated. This has revealed the energy management strategy and fuel economy of the ENVIRO $400 \mathrm{H}$.

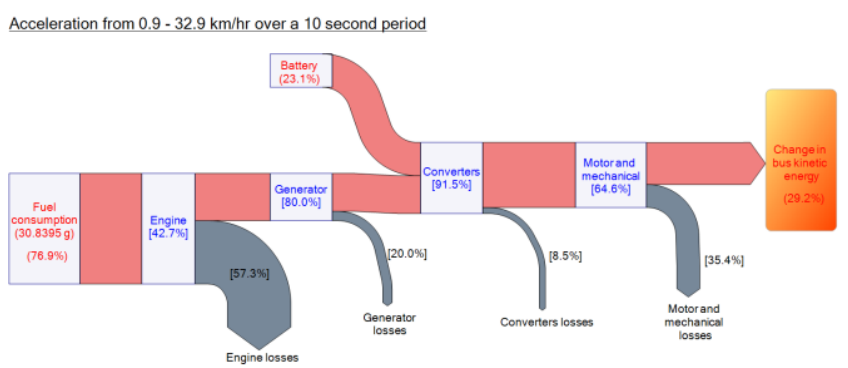

Figure 8 Sankey diagram describing acceleration maneuverer in 10 seconds period on flat surface.

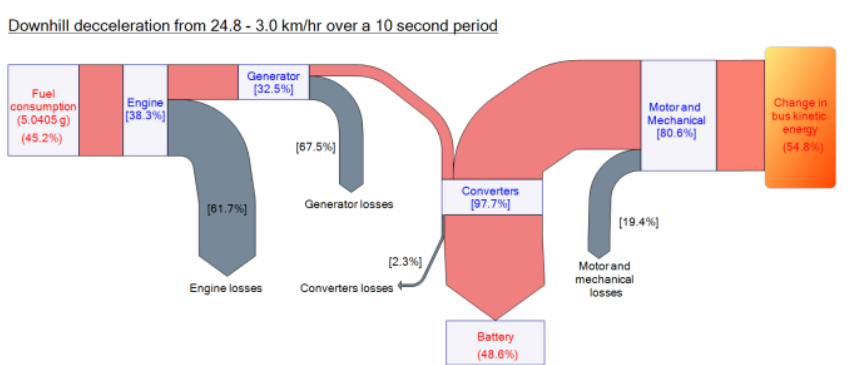

Figure 9 Sankey diagram describing deceleration maneuverer in 10 seconds period downhill.

After observing numerous periods of performance data, several general findings were observed. It was found that a fuel economy of $8.8 \mathrm{mpg}$ was found when the bus was operating on route 388 and $7.3 \mathrm{mpg}$ for the special route. These are slightly better than the $7.2 \mathrm{mpg}$ reported by TfL for the same bus in operation in London. This may be due to a number of reasons, such as the low passenger count observed for the collected data or the flat nature of the route. Further analysis is being carried out to determine the impact of different scenarios such as road gradient and traffic conditions on the fuel economy. In addition, it was found that State-of-Charge of the battery is normally maintained at around $40 \%$ with an overall variation between $30 \%-44 \%$. The reason for maintaining a small range of State-of-Charge is related to ease the charge/discharge 
columbic efficiencies to increase battery efficiency and life extension [6]. Another important finding is the significant losses from engine idling at $725 \mathrm{rpm}$, when fuel is consumed without the generator delivering electricity. This has a detrimental impact on the fuel economy and the overall impact is yet to be determined. The reason for this is the coupling between the engine and generator disconnects at low speeds to prevent stalling. Although engine speed is considered to be variable, when it comes to efficiency the engine could also be run constantly at $1500 \mathrm{rpm}$ to maximize efficiency [10], the impact of such an operating strategy on fuel economy is being investigated. Auxiliary power is negligible due to its magnitude being less than $1 \%$ when compared to other components in the system. In addition, regenerative energy contributes significantly to the battery SoC.

\section{COMPUTER MODEL}

Early stage hypothetical models were constructed in Simulink of various hybrid drive trains and included diesel engine, battery, supercapacitor, and fuel cell technologies.

The data presented in the previous sections provide large amounts of useful data to help in comparing the various computer models. The idea is to construct the exact diesel electric hybrid bus model of the ENVIRO $400 \mathrm{H}$ and simulate a bus-load model to simulate real road conditions. With the aid of the bus performance data and determined energy management strategy, this particular model can be the foundation with which to compare the proposed fuel cell hybrid models. The idea is to replace the diesel engine with a fuel cell and put suitable energy storage systems into the propulsion system. The aim is to reduce the emissions and study the feasibility of cost effective fuel cell hybrid bus designs based on the fuel economy research in the previous sections.

\section{HYDROGEN BUS}

Hydrogen buses have a fuel cell on board as the major energy source. They are emission free with the only by-product being water vapour. The problem is their cost has been prohibitively high with the most costly component being the fuel cell. Even though fuel cell prices have dropped significantly in the last decade, they are still incomparable to conventional diesel bus prices. For example, a diesel bus costs $£ 200,000$, a diesel electric hybrid bus costs $£ 300,000$, and one fuel cell bus cost $£ 2.5$ million including infrastructure in UK. In London, there are hydrogen buses operating on the RV1 route. The bus itself is a single decker bus, with series hybrid propulsion system using a $200 \mathrm{~kW}$ fuel cell, supercapacitor ESS, and power electronics [11]. The route it operates on is relatively flat and short so it is not challenging. On the other hand, the United States has a fuel cell bus fleet project which has a very similar fuel cell hybrid design and bus layout i.e. single decker bus, Ballard HD6 150kW fuel cell, BAE Systems hybrid system [12]. The main difference is that the RV1 adopts supercapacitors as the ESS, whereas the US hydrogen bus adopts Li-ion batteries as the ESS. The problems causing bus unavailability are also highly similar, e.g. power electronics break down, fuel cell malfunction. The fuel cell plant lifetime is $660-20,000$ hours; the buses cost $\$ 2,100,000-2,400,000$ each; the fuel economy is 5.56 to 7.71 miles per diesel gallon equivalent (DGE) so far and so reach the DoE target in 2016 of 8 DGE [13].

\section{FUTURE WORK}

The data collection and processing has been completed, with the selection of scenarios and behaviours to be analysed being finalised. The impact on fuel economy of different scenarios and parameters, such as passenger weight and traffic conditions need to be explored. Some minor issues can be resolved if there is enough time, e.g. complete video synchronisation. The focus will then be on the computer modelling and concern validation of the diesel hybrid model with the performance of a bus based on the collected data and energy management strategy. This will be used as the benchmark with which to compare the performance of the different FC hybrid propulsion systems developed in Simulink. These will examine future improvements on new designs for a fuel cell bus for London. Computer modelling of possible FC hybrid configurations and component sizing will be used to determine the viability of FC hybrid drive trains for city driving buses. This study will be presented in the future and focus on the fuel economy and effective design for cost reduction.

\section{CONCLUSION}

In this study, the performance of a diesel electric hybrid bus has been analysed through the collection and analysis of realworld performance data. The data was obtained from multiple sources, then synchronised, processed and presented using a MATLAB program. The results indicate a fuel economy of between 7.3 to $8.8 \mathrm{mpg}$ for the diesel hybrid bus operating in London. In addition the operating strategy of the drive system has been determined. Finally the operational profile has been determined and can be used as the benchmark to test and compare the performance of different drive trains, with the aim of developing a FC hybrid system that is capable of meeting the performance requirements of city driving.

\section{ACKNOWLEDGEMENTS}

Thanks to BAE Systems providing huge resources and help. Thanks to Dr. David Ashmore who made the experiments happened.

HyFCap joint project EPSRC grant number 0/K021192/1

\section{REFERENCES}

[1] "IEA - International Energy Agency." [Online]. Available: http://www.iea.org/.

[2] "National Travel Survey statistics - GOV.UK." [Online]. Available: https://www.gov.uk/government/collections/nationaltravel-survey-statistics..

[3] T. for London, "Home." [Online]. Available: http://www.tfl.gov.uk/.

[4] L. Kumar and S. Jain, "Electric propulsion system for electric 
vehicular technology: A review," Renew. Sustain. Energy Rev., vol. 29, pp. 924-940, Jan. 2014.

[5] Q. Cai, D. J. L. Brett, D. Browning, and N. P. Brandon, “A sizingdesign methodology for hybrid fuel cell power systems and its application to an unmanned underwater vehicle," J. Power Sources, vol. 195, no. 19, pp. 6559-6569, Oct. 2010.

[6] A. González, E. Goikolea, J. Andoni, and R. Mysyk, "Review on supercapacitors : Technologies and materials," vol. 58, pp. 11891206, 2016.

"Welcome to Alexander Dennis." [Online]. Available: http://www.alexander-dennis.com/.

[8] “Google Maps API | Google Developers.” [Online]. Available: https://developers.google.com/maps/.

[9] "ISO - International Organization for Standardization." [Online]. Available: http://www.iso.org/iso/home.html.

[10] "Engine Performance Curve ISB4.5 186 P7-0 @ 2011, Cummins Inc.," 2011.

[11] "The Clean Hydrogen in European Cities (CHIC)." [Online]. Available: http://chic-project.eu/.

[12] L. Eudy and M. Post, "Fuel Cell Buses in U.S. Transit Fleets: Current Status 2015,” no. December, 2015.

[13] "Department of Energy Washington DC." [Online]. Available: http://www.energy.gov/. 